\title{
Acceptance of Parents to Silver Diamine Fluoride in Pre-cooperative Children: A Cross-sectional Study
}

\author{
Pooja R Shivasharan ${ }^{1}$, Ashwin M Jawdekar², Neha N Pankey ${ }^{3}$, Surej Unnikrishnan ${ }^{4}$
}

\begin{abstract}
Aim: To evaluate the acceptance of silver diamine fluoride (SDF) among parents of pre-cooperative children.

Materials and methods: This was a questionnaire-based study. Prevalidated questionnaires were distributed to the parents of pre-cooperative children with dental caries. The response rate was $100 \%$. Data were statistically analyzed using Chi-square test.

Results: Of 105 parents, $71.42 \%$ were mothers, $21.90 \%$ were fathers, and $6.66 \%$ were other caregivers. In relation to noncooperation of the children in the past, $22.85 \%$ of the parents of the children above 36 months chose SDF, whereas only $4.76 \%$ of the parents of children 36 months of age and below chose SDF ( $p=0.047$ ). Discoloration of front teeth was acceptable to $33.33 \%$ of the parents below the income of 25,000 INR and was not acceptable to $14.29 \%(p=0.033)$. For front teeth, $33.33 \%$ of the parents above the income of 25,000 INR did not accept SDF treatment when there were behavioral barriers, whereas $13.33 \%$ accepted it $(p=0.003)$. Also, acceptance of SDF for back teeth was $32.38 \%$ to the parents below the income of 25,000 INR in contrast to $15.24 \%$ of who did not accept it $(p=0.001)$.

Conclusion: SDF is acceptable to parents and potentially useful in patients who are unable to obtain traditional restorative treatments due to cooperation, access, costs, and apprehension of general anesthesia.
\end{abstract}

Keywords: Dental caries, Esthetic concerns, Parental acceptance, Pre-cooperative children, Silver diamine fluoride.

Journal of South Asian Association of Pediatric Dentistry (2019): 10.5005/jp-journals-10077-3025

\section{INTRODUCTION}

In a developing country like India, dental caries is seen to be widely prevalent and severe. In a recent systematic review, the prevalence of early childhood caries in India was $41.49 \%{ }^{1}$ In another review by Mehta, one out of two children in India were affected by dental caries. ${ }^{2}$ The overall caries prevalence in primary school children of India in a cross-sectional study was seen to be $78.9 \% .^{3}$ In children, dental anxiety and fear of dental treatment have been recognized as problems in management for many years, which can affect the quality of care. ${ }^{4}$ In a survey (Vellingiri), parent's preference to general or local anesthesia for their children's dental treatments was evaluated. Ninety-seven percent of the parents preferred local anesthesia to general anesthesia. ${ }^{5}$

Silver diamine fluoride (SDF) is a topical agent used in many countries to arrest dental caries. It has dual mechanism of action as the silver component acts as an antimicrobial agent by killing the bacteria and preventing the formation of new biofilm, whereas the fluoride acts by preventing further demineralization of tooth structure. ${ }^{6,7}$ SDF is considered to be cost effective, easy to perform, and noninvasive technique for caries management. It can be used to slow down or arrest the caries progression in very young children. In addition, it can be used for disadvantaged children or in areas where there is great shortage of dental personnel. ${ }^{8}$ Also, it was cleared as a desensitizing agent by the Food and Drug Administration for the US market in 2014. ${ }^{9}$ According to Mellberg et al., fluoride introduced into the oral cavity gets cleared with passage of time; hence, a continuous supply of fluoride is essential for anticaries effect. ${ }^{10}$ Enamel fluoride content was seen to increase significantly even after 6 months of application of SDF compared to fluoride varnish and APF gel. ${ }^{11}$ A systematic review of seven studies indicated that SDF, at concentrations of $30 \%$ and $38 \%$, was more effective than other preventive management strategies for arresting dentinal caries in the primary dentition. ${ }^{12} \mathrm{~A}$ meta-analysis performed on extracted data from 8 studies using $38 \%$

\footnotetext{
${ }^{1-4}$ Department of Paedodontics, Dr GD Pol Foundation's YMT Dental College and Hospital, Navi Mumbai, Maharashtra, India
}

Corresponding Author: Pooja R Shivasharan, Department of Paedodontics, Dr GD Pol Foundation's YMT Dental College and Hospital, Navi Mumbai, Maharashtra, India, Phone: +91 9960622964 , e-mail: dr.poojashivasharan@gmail.com

How to cite this article: Shivasharan PR, Jawdekar AM, Pankey NN, et al. Acceptance of Parents to Silver Diamine Fluoride in Pre-cooperative Children: A Cross-sectional Study. J South Asian Assoc Pediatr Dent 2019;2(2):49-55.

Source of support: Nil

Conflict of interest: None

SDF to arrest caries in primary teeth reported the overall percentage of active caries that became arrested to be $81 \% .^{13}$

Despite these benefits, SDF is known to cause dark staining of carious enamel and dentin which can result in esthetic concerns among parents. Owing to the scarcity of literature about SDF in Indian context regarding its acceptance to the possibility of esthetic concerns and other aspects among parents, we planned a study with the following main objectives:

- Parental acceptance of SDF treatment in pre-cooperative children with limited cooperation.

- Parental acceptance of SDF treatment in view of possible discoloration of back and/or front teeth.

We also evaluated the impact of modifying factors such as parent's income, education status, gender, age, etc.

\section{Materials and Methods}

This was a questionnaire-based cross-sectional study. The study was conducted in the Department of Paediatric and Preventive Dentistry 
of teaching institute in India. Parents of the patients attending the OPD were selected. Ethical clearance for the study was obtained from the Institutional Ethical Committee. A written consent was obtained from each parent(s) of children prior to commencing the study. We determined that a sample of at least 96 study participants to detect a difference of 0.2 standard deviations with error rate of $5 \%$ using the formula $n=Z \alpha / E$. However, we included 105 participants for this study to account for errors, if any, owing to improperly filled questionnaires or participant dropouts from the study.

Parents of pre-cooperative children who have visited the dentist to seek treatment for carious teeth and children having at least one carious lesion involving dentin which is soft, yellow in color and painless were selected for the study. Children with painful and abscessed teeth and those with developmental defects (amelogenesis imperfecta, dentinogenesia imperfecta) were excluded from the study.

Questionnaire used was a modified version of the one used by Crystal. ${ }^{14}$ The same was revalidated by two experts in the department. Also, a pilot study on five patients was done. The questionnaire was assessed under four domains. First part of which consisted of the demographic details of the parent and the child, second part consisted of past dental experience of the child, third part consisted of acceptance of discoloration by the parents in case of different behaviors of the child, and fourth part consisted of acceptance of discoloration by the parents in case pharmacological methods were required to complete the fillings. Photographs of SDF before and after treatment for both front and back teeth were included in the questionnaire to evaluate acceptance to esthetics by the parents (Figs 1 and 2). A study tool comprising this questionnaire was administered to each participant. Response rate was $100 \%$.

The data were organized using MS Excel program and subjected to statistical analysis.

\section{Results}

The demographic details of the participants responding to the survey are outlined in Table 1. About forty-seven percent of the caregivers were between the age group of 21 years and 30 years, $42.9 \%$ were in the age group of $31-40$ years, $4.8 \%$ were in the age group of $41-50$ years, and only $5.7 \%$ were in the age group of 50 years and above. Out of total 105 parents, $71.42 \%$ were mothers,
$21.90 \%$ were fathers, and $6.66 \%$ were other caregivers. The mean income of the caregivers was 42321.21 ( \pm SD 39782.511) INR. 5.7\% of caregivers never attended school, $1.9 \%$ studied up to primary school, $16.2 \%$ studied up to secondary school, $12.4 \%$ studied up to 10th standard, $19.0 \%$ studied up to 12 th standard, $24.8 \%$ graduated from college, and $20.0 \%$ postgraduated from college. The mean age of the children selected for this study was 41.19 months ( \pm SD 9.19). Out of these, $53.3 \%$ were boys and $46.7 \%$ were girls. When asked about the baby teeth, $75.23 \%$ of the caregivers stated that baby teeth were important to them.

The correlation between parents choosing SDF in relation to different variables like noncooperation of child in the past, discoloration, behavioral barriers, and pharmacological methods is reported in Table 2. In relation to noncooperation of the children in past, $22.85 \%$ of the parents of the children above 36 months chose SDF whereas only $4.76 \%$ of the parents of children 36 months of age and below were seen to choose SDF (Fig. 3). This difference was statistically significant ( $p=0.047$ ).

The correlation between acceptance of SDF in front teeth and back teeth and the relative modifying factors are depicted in Table 3.

The discoloration caused by SDF was acceptable to $38.1 \%$ and $60 \%$ of mothers w.r.t. front and back teeth, respectively, whereas it was acceptable to $15.24 \%$ and $18.1 \%$ of fathers w.r.t. front and back teeth, respectively. This finding was statistically insignificant $(p>0.05)$.

For front teeth, the discoloration caused by SDF was acceptable to $27.62 \%$ of the parents aged between 21 years and 30 years, $22.86 \%$ of the parents aged between 31 years and 40 years, $3.81 \%$ of the parents aged between 41 years and 50 years, and $4.76 \%$ of the parents aged 50 years and above. This difference was statistically insignificant $(p=0.395)$.

For back teeth, the discoloration caused by SDF was acceptable to $39.05 \%$ of the parents aged between 21 years and 30 years, $35.24 \%$ of the parents aged between 31 years and 40 years, $4.76 \%$ of the parents aged between 41 years and 50 years, and $5.71 \%$ of the parents aged 50 years and above. This difference was statistically insignificant ( $p=0.523)$.

The discoloration of front teeth was acceptable to $33.33 \%$ of the parents below the income of 25,000 INR and was not acceptable to $14.29 \%$. This value was statistically significant $(p=0.033$ ) (Table 3 and Fig. 4).
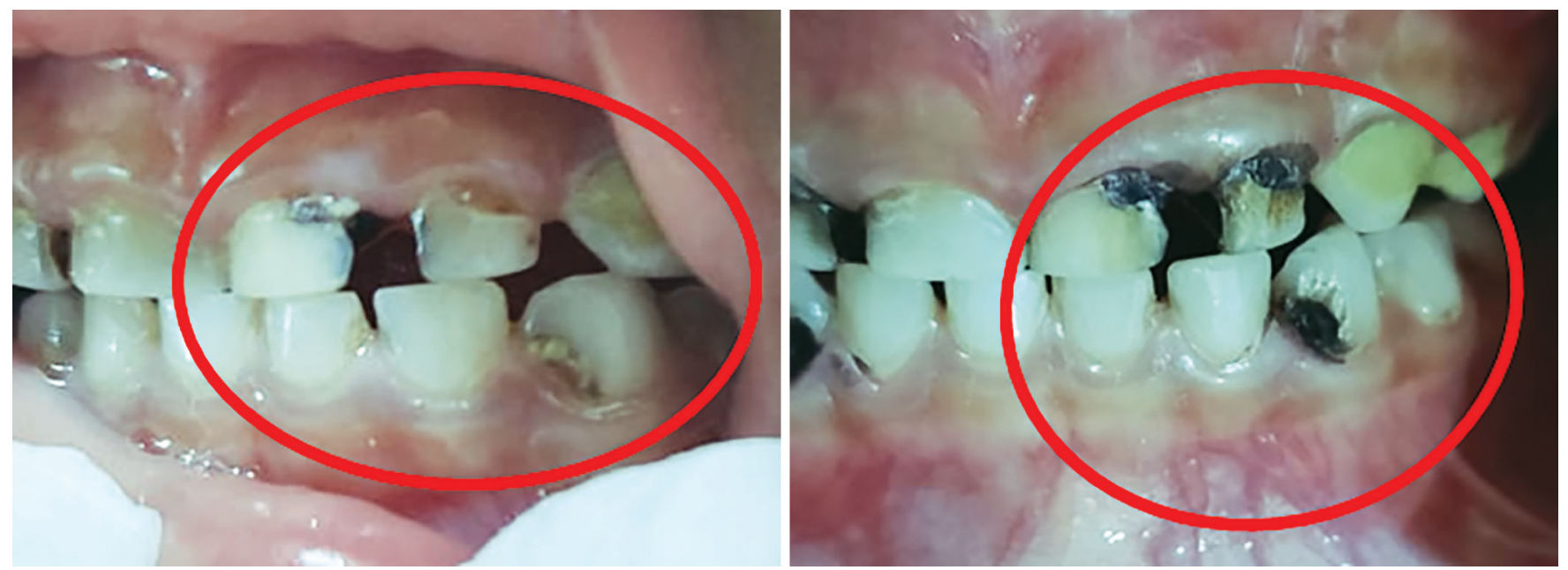

Fig. 1: Discoloration associated with SDF in front teeth: (left) cavitated lesion before treatment with SDF and (right) cavitated lesion after treatment with SDF 

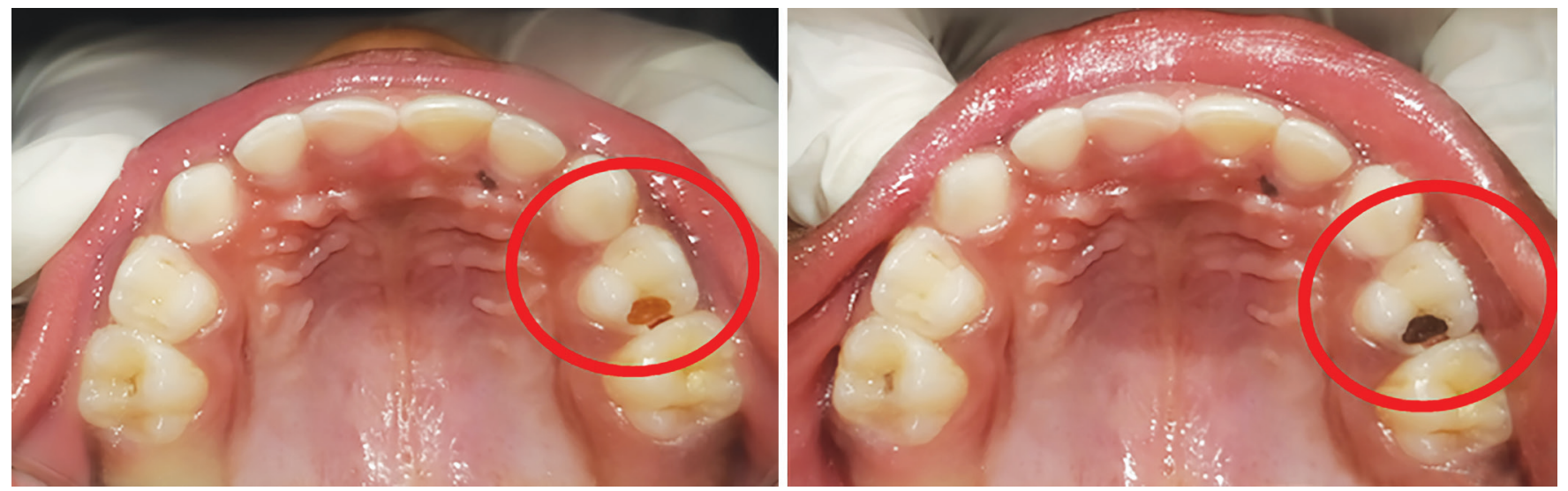

Fig. 2: Discoloration associated with SDF in back teeth: (left) cavitated lesion before treatment with SDF and (right) cavitated lesion after treatment with SDF

Table 1: Characteristics of study population

\begin{tabular}{ll}
\hline Variables & Distribution \\
\hline Age of the child in months & Mean \pm SD $41.19 \pm 9.19$ \\
Caregiver's age in years & $46.7 \%(21-30) 42.9 \%(31-40) 4.8 \%(41-50) 5.7 \%(>50)$ \\
Caregiver type & $71.4 \%$ (mother) $21.9 \%$ (father) $4.8 \%$ (other female) $1.9 \%$ (other male) \\
Income & Mean \pm SD $42321.21 \pm 39782.511$ \\
Education category & $5.7 \%$ (did not attend school) $1.9 \%$ (primary school) $16.2 \%$ (secondary \\
& school) $12.4 \%$ (10th pass) $19.0 \%$ (12th pass) $24.8 \%$ (graduation from \\
Gender of the children & college) $20.0 \%$ (postgraduation from college) \\
Gender of the caregivers & $53.3 \%$ (boys) $46.7 \%$ (girls) \\
Baby teeth importance & $23.80 \%$ (male) $76.19 \%$ (female) \\
\hline
\end{tabular}

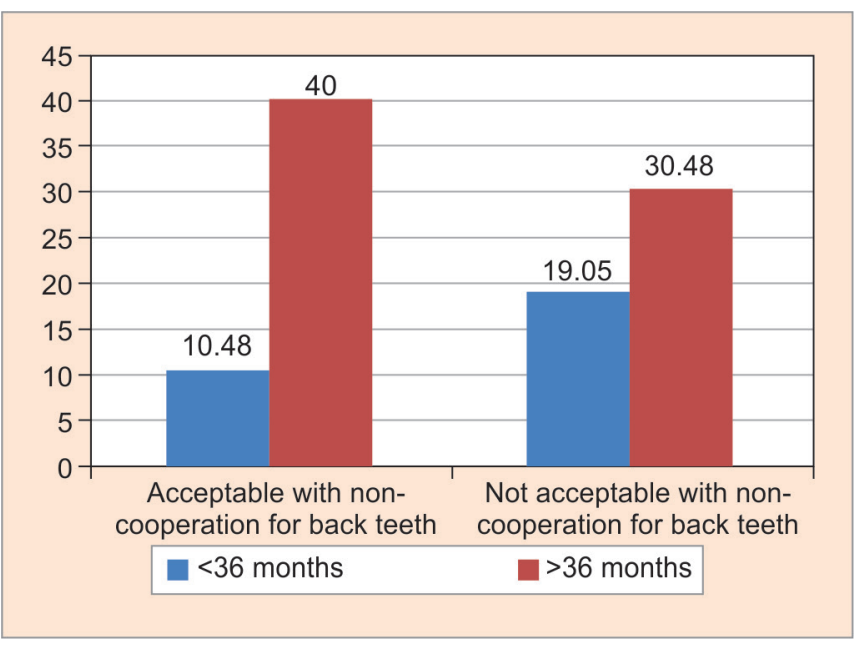

Fig. 3: Acceptability of SDF with noncooperative children above and below the mean age for back teeth

If children showed noncooperation for restorations, $29.52 \%$ of mothers and $9.52 \%$ of fathers would choose SDF in front teeth whereas $35.24 \%$ of mothers and $10.48 \%$ of fathers would choose SDF in back teeth $(p>0.05)$.

For front teeth, $33.33 \%$ of the parents above the income of 25,000 INR did not accept SDF treatment when there were behavioral barriers whereas $13.33 \%$ accepted it. This value was statistically highly significant ( $p=0.003$ ) (Table 3 and Fig. 5).

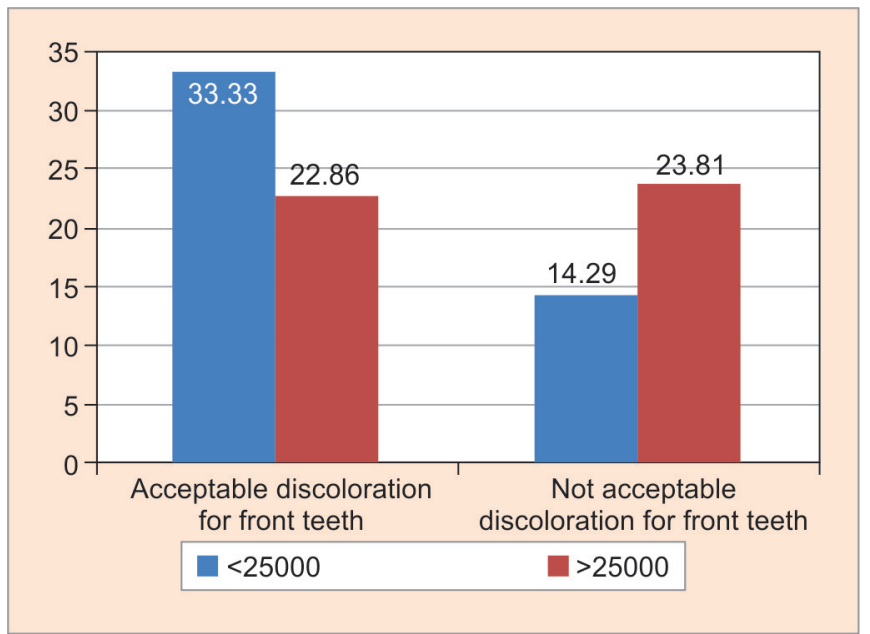

Fig. 4: Acceptability of SDF with parents above and below the mean income for discoloration of front teeth

Also, acceptance of SDF for back teeth was $32.38 \%$ in the parents below the income of 25,000 INR contrast to $15.24 \%$ of the parents who did not accept it (Table 4 and Fig. 6). This value was statistically highly significant ( $p=0.001)$.

When education status was considered, $29.5 \%$ of the parents of low education status would choose in front teeth if the child showed uncooperative behavior. Conversely, 31.4\% of the parents of high education status would not choose SDF in front teeth if the 


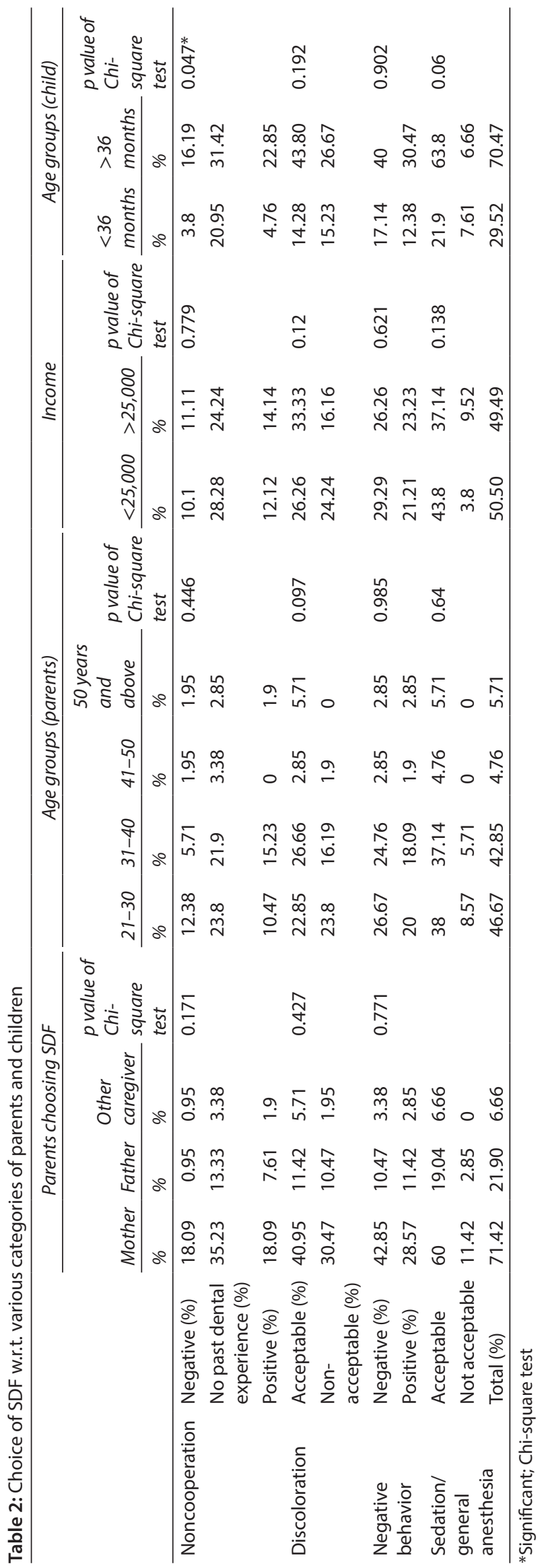




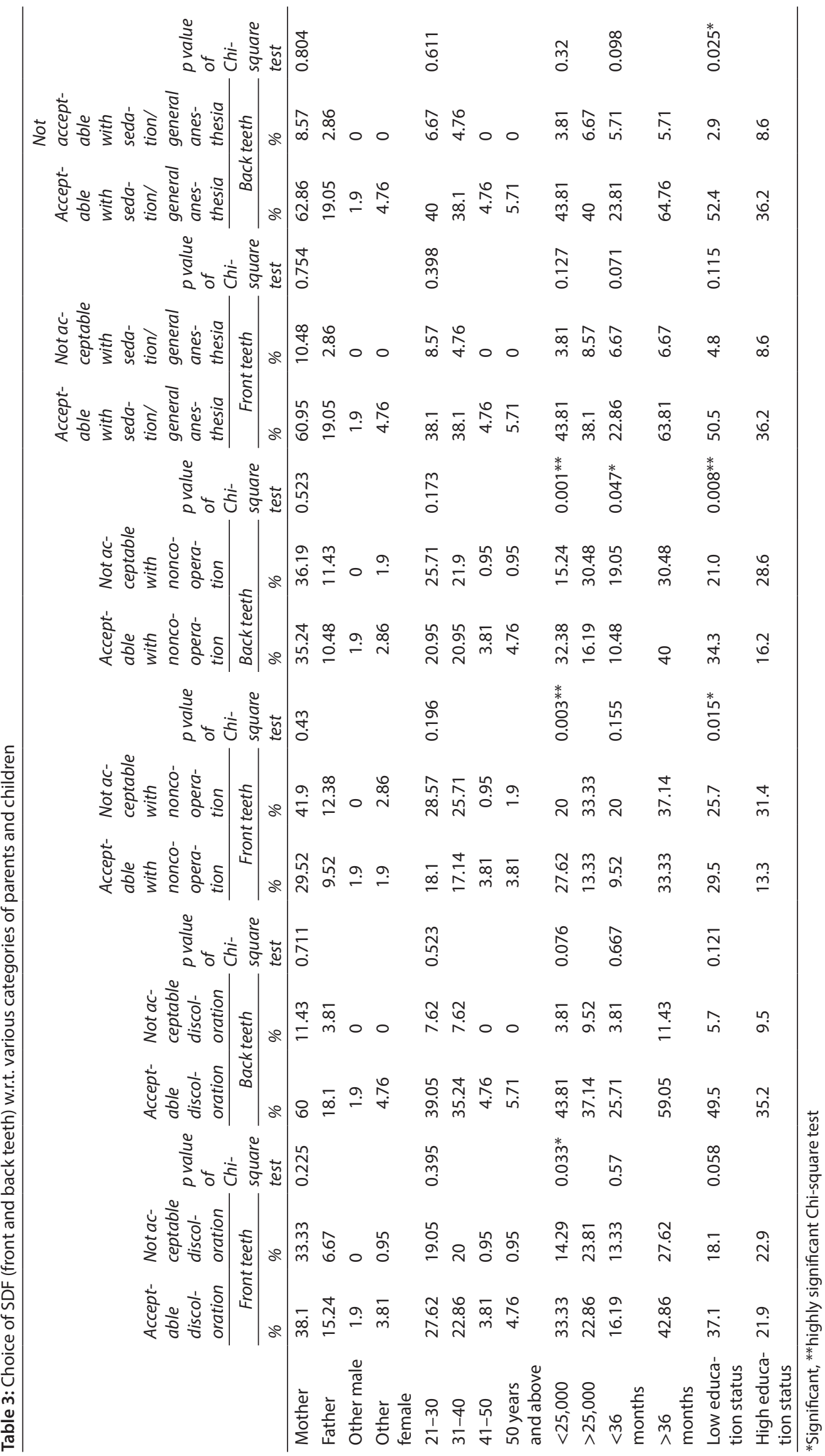


Acceptance of Parents to Silver Diamine Fluoride in Pre-cooperative Children: A Cross-sectional Study

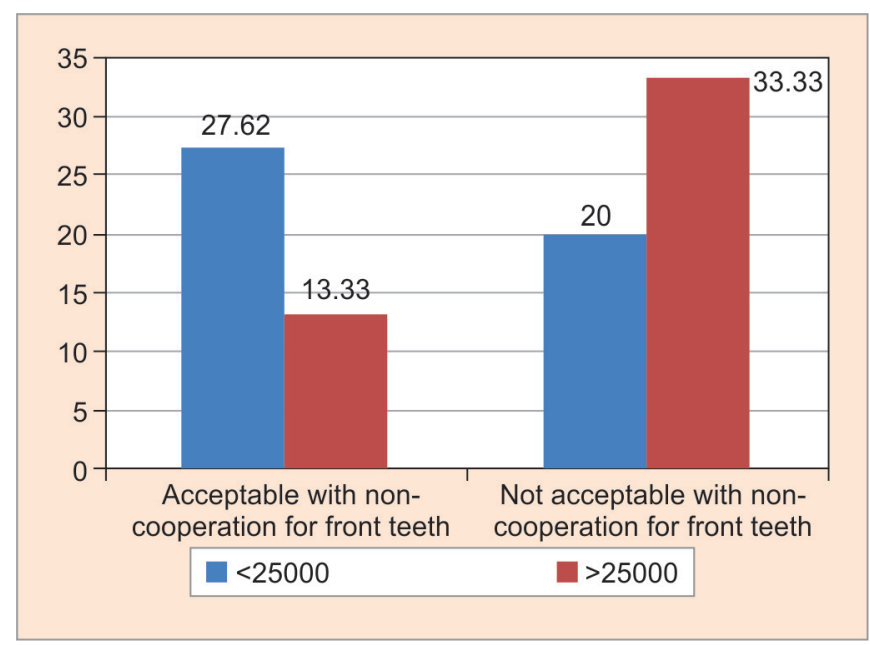

Fig. 5: Acceptability of SDF for front teeth in parents above and below mean income in noncooperative children

child showed uncooperative behavior. This value was statistically significant ( $p=0.015)$.

Also, 34.3\% of the parents of low education status would choose SDF in back teeth when child showed uncooperative behavior. However, $28.6 \%$ of the parents of high education status would not choose SDF in back teeth if child showed uncooperative behavior. This value is statistically significant $(p=0.008)$.

In addition, $52.4 \%$ of parents of low education status would choose SDF instead of general anesthesia or sedation whereas $8.6 \%$ of parent of high education status would not choose SDF but would opt for restorative treatment under general anesthesia or sedation $(p=0.025)$.

\section{Discussion}

The present study being a cross-sectional study mainly focuses on acceptance of parents to SDF staining in pre-cooperative children. The study included 105 parents of children with at least one carious lesion. To our knowledge, this is the first ever study done on the Indian population with the above mentioned study objective. In relation to noncooperation of the child in the past, the percentage of parents accepting SDF was more in the children above 36 months, which may be due to no past dental experience in most of the children below 36 months.

In our study, acceptance to SDF by parents was seen to be far more for back teeth than for the front teeth. This difference may be because of the less visibility of discoloration in the back teeth when compared to the front teeth. These results were in conjunction to the study done by Crystal ${ }^{14}$ and Bagher. ${ }^{15}$ However, contrasting results were reported by Alshammari ${ }^{16}$ where majority of the parents strongly refused SDF treatment both in front and back teeth. Also, other authors have reported that parents preferred tooth-colored restorations over amalgams and silver metal crowns ${ }^{17}$ and zirconia esthetic crowns over other anterior restorations. ${ }^{18}$

Discoloration of front teeth was one of the major concerns among most of the parents. The investigators in a study concluded that the degree of dentin demineralization led to a significant increase in the rate of dentin color change after application of SDF. ${ }^{19}$

However, a significant number of parents below the mean income accepted SDF discoloration in front teeth when compared to the parents with income above it. This can be due

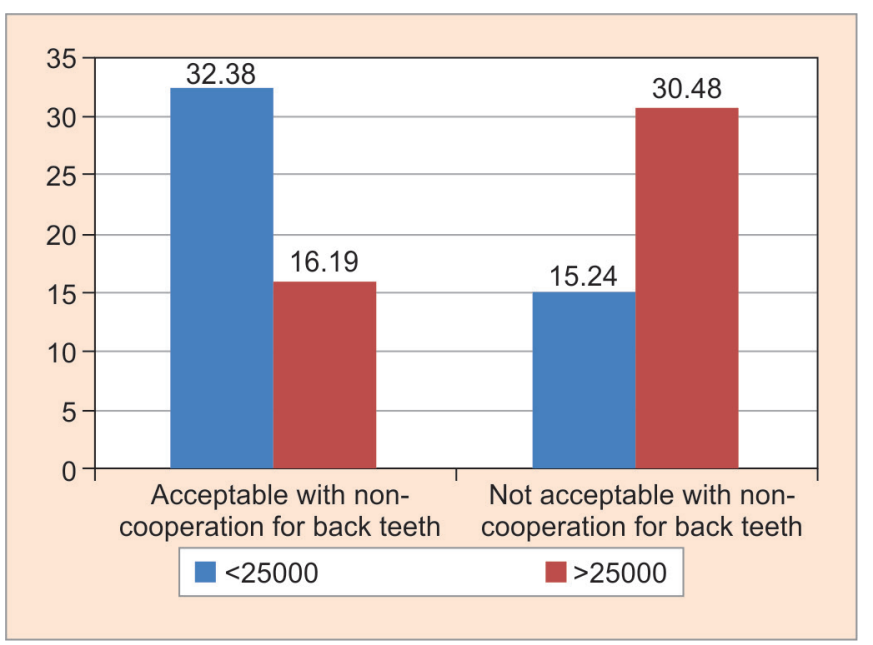

Fig. 6: Acceptability of SDF for back teeth in parents above and below mean income in noncooperative children

to the superficial or partial knowledge and lack of awareness among the parents about importance of primary teeth w.r.t. esthetics. $^{20}$

Although discoloration caused by SDF was unacceptable to most of the parents, many would still choose SDF instead of pharmacological methods like sedation or general anesthesia. These results were similar to the study done by Bagher, ${ }^{15}$ wherein parents of children with history of uncooperative behavior during previous dental treatment were significantly accepting SDF regardless of type and location of teeth. The reason for choosing SDF may be the parent's awareness of anesthetic agents being associated with potential risk to patient's overall health with some reports of morbidity and mortality. ${ }^{21,22}$

In our analysis, we found that majority of parents of both low and high education status would choose SDF instead of general anesthesia and sedation. However, results reported by Crystal ${ }^{14}$ showed similar results with no difference in acceptance of SDF when parents had to consider the need for general anesthesia.

In our study, preoperative and postoperative pictures of SDF treatment were added in the questionnaire which could give a clear idea of discoloration to the parents. It is recommended that the consent forms with photographs of SDF application on front and back teeth should be presented to the parents before SDF application. $^{15}$

In recent literature, SDF has attracted attention as an additional approach to caries management. It is bactericidal to cariogenic bacteria mainly Streptococcus mutans, promotes remineralization of demineralized enamel and dentine, and also hampers degradation of the dentine collagen. ${ }^{23}$ In view of the findings in the systematic review; SDF was a more effective dentine caries arresting agent than sodium fluoride. ${ }^{24}$ Also, in another study, it was effective in arresting active caries lesions in primary teeth in young children and was well accepted by parents. ${ }^{25}$

There were certain limitations associated with the current study. This study was limited to the parents who had visited the said institution for treatment purpose with their children. Cost was not a parameter considered for the acceptance of SDF as the treatments provided in the college are free. Lastly, most of the children had no past dental experience which might have affected the choice of SDF by the parents. 


\section{CONCLUSION}

SDF can be considered acceptable to parents and is potentially useful in patients who are unable to obtain traditional restorative treatments due to cooperation, access, costs and apprehension of general anesthesia. Large multicentric studies in public as well as private setups are necessary to substantiate the claims made by this study.

\section{References}

1. Ganesh A, Muthu MS, Mohan A, et al. Prevalence of early childhood caries in India - a systematic review. Indian J Pediatr 2019;86(3): 276-286. DOI: 10.1007/s12098-018-2793-y.

2. Mehta A. Trends in dental caries in Indian children for the past 25 years. Indian J Dent Res 2018;29(3):323-328. DOI: 10.4103/ijdr. IJDR_615_17.

3. Hiremath A, Murugaboopathy V, Ankola AV, et al. Prevalence of dental caries among primary school children of India - a crosssectional study. J Clin Diagn Res 2016;10(10):ZC47-ZC50. DOI: 10.7860/ JCDR/2016/22474.8642.

4. Sharma A, Tyagi R. Behaviour assessment of children in dental settings: a retrospective study. Int J Clin Pediatr Dent 2011;4(1):35-39. DOI: 10.5005/jp-journals-10005-1078.

5. Vellingiri S, Gurunathan D. Assessment of parent's preference to general or local anesthesia for children undergoing dental treatment. World J Dent 2015;6(3):154-160. DOI: 10.5005/jp-journals-10015-1333.

6. Yee R, Holmgren C, Mulder J, et al. Efficacy of silver diamine fluoride for arresting caries treatment. J Dent Res 2009;88(7):644-647. DOI: 10.1177/0022034509338671.

7. $\mathrm{Chu} \mathrm{CH}$, Lo HC, Lin HC. Effectiveness of silver diamine fluoride varnish in arresting dentin caries in Chinese preschool children. J Dent Res 2002;81(11):767-770. DOI: 10.1177/0810767.

8. $\mathrm{Chu} \mathrm{CH}$, Lo ECM. Promoting caries arrest in children with silver diamine fluoride: a review. Oral Health Prev Dent 2008;6(4):315-321.

9. Ngoc CN, Mehta R, Donovan TE, et al. Teaching silver diamine fluoride in U.S. dental school's predoctoral curricula. J Dent Educ 2018;82(12):1305-1309. DOI: 10.21815/JDE.018.141.

10. Mellberg JR, Ripa LW, Leske GS Fluoride in Preventive dentistry Theory and Clinical Applications Chicago: Quintessence Publishing Co; 1983.

11. Shah SG, BhaskarV, Chawla S, et al. Efficacy of silver diamine fluoride as a topical fluoride agent compared to fluoride varnish and acidulated phosphate fluoride gel: an in vivo study. J Pediatr Dent 2014;2(1):5-12. DOI: 10.4103/2321-6646.130376.
12. Contreras V, Toro MJ, Elías-Boneta AR, et al. Effectiveness of silver diamine fluoride in caries prevention and arrest: a systematic literature review. Gen Dent 2017;65(3):22-29.

13. Gao SS, Zhao IS, Hiraishi N, et al. Clinical trials of silver diamine fluoride in arresting caries among children: a systematic review. JDR Clin Trans Res 2016;1(3):201-210. DOI: 10.1177/2380084416661474.

14. Crystal YO, Janal MN, Hamilton DS, et al. Parental perceptions and acceptance of silver diamine fluoride staining. J Am Dent Assoc 2017;148(7):518-518. DOI: 10.1016/j.adaj.2017.03.013.

15. Bagher SM, Sabbagh HJ, Aljohani SM, et al. Parental acceptance of the utilization of silver diamine fluoride on their child's primary and permanent teeth. Patient Prefer Adherence 2019;13:829-835. DOI: 10.2147/PPA.S205686.

16. Alshammari AF, Almuqrin AA, Aldakhil AM, et al. Parental perceptions and acceptance of silver diamine fluoride treatment in Kingdom Saudi Arabia. Int J Health Sci (Qassim) 2019;13(2):25-29.

17. Holan G, Rahme MA, Ram D. Parents' attitude toward their children's appearance in the case of esthetic defects of the anterior primary teeth. J Clin Pediatr Dent 2009;34(2):141-145. DOI: 10.17796/jcpd.34.2. 910037663v7pm6vg.

18. Pani SC, Saffan AA, AlHobail S, et al. Esthetic concerns and acceptability of treatment modalitiesin primary teeth: a comparison between children and their parents. Int J Dent 2016;2016:3163904. DOI: 10.1155/2016/3163904

19. Sayed M, Matsui N, Hiraishi N, et al. Evaluation of discolouration of sound/demineralised root dentin with silver diamine fluoride: in vitro study. Dent Mater J 2019;38(1):143-149. DOI: 10.4012/dmj.2018-008.

20. Setty JV, Srinivasan I. Knowledge and awareness of primary teeth and their importance among parents in Bengaluru city, India. Int J Clin Pediatr Dent 2016;9(1):56-61. DOI: 10.5005/jp-journals-10005-1334.

21. Thikkurissy S, Crawford B, Groner J, et al. Effect of passive smoke exposure on general anaesthesia for pediatric dental patients. Anesth Prog 2012;59(4):143-146. DOI: 10.2344/0003-3006-59.4.143.

22. Malhotra N. General anaesthesia for dentistry. Indian J Anaesth 2008;52(Suppl 5):725-737.

23. Zhao IS, Gao SS, Hiraishi N, et al. Mechanisms of silver diamine fluoride on arresting caries: a literature review. Int Dent J 2018;68(2):67-76. DOI: $10.1111 /$ idj.12320.

24. Trieu A, Mohamed A, Lynch E. Silver diamine fluoride vs sodium fluoride for arresting dentine caries in children: a systematic review and meta-analysis. Sci Rep 2019;9(1):2115. DOI: 10.1038/s41598-01938569-9.

25. Clemens J, Gold J, Chaffin J. Effect and acceptance of silver diamine fluoride treatment on dental caries in primary teeth. J Public Health Dent 2018;78(1):63-68. DOI: 10.1111/jphd.12241. 